Article

\title{
The Pre-Potential of a Field Propagating with the Speed of Light and Its Dual Symmetry
}

\author{
Yaakov Friedman $1, *(1)$, David Hai Gootvilig ${ }^{2}$ and Tzvi Scarr ${ }^{3}$ (1) \\ 1 Extended Relativity Research Center, Departments of Mathematics and Physics, Jerusalem College of \\ Technology, P.O. Box 16031, Jerusalem 91160, Israel \\ 2 Department of Physics, Jerusalem College of Technology, P.O. Box 16031, Jerusalem 91160, Israel; \\ gootvilig.davidhai@gmail.com \\ 3 Department of Mathematics, Jerusalem College of Technology, P.O. Box 16031, Jerusalem 91160, Israel; \\ scarr@g.jct.ac.il \\ * Correspondence: friedman@g.jct.ac.il
}

Received: 10 October 2019; Accepted: 18 November 2019; Published: 20 November 2019

check for updates

\begin{abstract}
Relativity theory assumes that force fields propagate with the speed of light. We show that such force fields generated by a single source can be described by a pre-potential, which is a complex-valued function on spacetime outside the worldline of the source. The pre-potential is invariant under a spin-half representation of the Lorentz group acting on complexified spacetime. The complex four-potential of such a field is defined and calculated explicitly from the pre-potential without assuming any particular force law for the field. The real part of the obtained four-potential coincides with the known Liénard-Wiechert potential. The symmetry of the four-potential is described herein. The pre-potential satisfies the wave equation. The single source electromagnetic field derived from this four-potential is self-dual or anti-self-dual. The pre-potential and the four-potential are extended to a field with several sources.
\end{abstract}

Keywords: retarded potential; Whittaker scalar potential; pre-potential; Lorentz invariance; spin-half representation; self-dual field

\section{Introduction and Motivation}

The main distinction between classical and relativistic physics is the speed with which fields propagate. Classically, the field acts instantaneously, while in relativity it is assumed that fields propagate with the speed of light. It is known that both electric fields and gravitational fields propagate with the speed of light. These fields are generated by moving charges and moving masses, respectively. We start here with the field generated by a single source. A description of a field generated by more than one source can be obtained by integration.

In this paper, we describe the field using a scalar-valued pre-potential. This approach has its origins in the work of E. T. Whittaker, who in 1904 introduced [1] two scalar potential functions. He was thus able to reduce the degrees of freedom of the electromagnetic field description to two. He showed that the electromagnetic field can be expressed in terms of the second derivatives of these functions. However, he was not able to find the covariance of his scalar potentials. H. S. Ruse [2] improved the result of Whittaker. A. O. Barut, S. Malin and M. Semon [3,4] presented another alternative formulation of classical electrodynamics in terms of a single scalar function.

In 2009, the first author and S. Gwertzman [5] showed that it is possible to use Whittaker's two scalar potentials as the real and complex parts of one complex-valued function $\psi(x)$ on Minkowski space, called the pre-potential of the electromagnetic field. Moreover, in [6,7], it was shown that this pre-potential is invariant under a spin-half representation of the Lorentz group. Thus, this 
pre-potential provides a covariant description of an electromagnetic field with minimal degrees of freedom. The electromagnetic field tensor is a contraction of the second derivatives of the pre-potential with the Dirac $\alpha$-matrices. The pre-potential is also relevant to the Aharonov-Bohm effect [8].

One of the motivations for using complex numbers to describe a field comes from the two types of acceleration that a field generates. Linear acceleration expresses the rate of change of the magnitude of the velocity, while rotational acceleration expresses the rate of change of the direction of the velocity. For example, an electric field $\mathbf{E}$ generates a linear acceleration, and a magnetic field $\mathbf{B}$ generates a rotational acceleration. Note that $\mathbf{E}$ is a vector, but $\mathbf{B}$ is a pseudo-vector, meaning that its sign depends on an arbitrary choice of orientation. In a gravitational field, the linear acceleration vector can be measured by an accelerometer, while a gyroscope measures the rotational acceleration, which is also represented by a pseudo-vector. In order to combine $\mathbf{E}$ and $\mathbf{B}$, we introduce a pseudo-scalar $i$, which changes sign upon a change of orientation. We multiply the pseudo-vector $\mathbf{B}$ by the pseudo-scalar $i$. Then, the resulting complex Faraday vector $\mathbf{F}=\mathbf{E}+i \mathbf{B}$ is independent of the choice of orientation.

The electromagnetic field strength tensor can be described as a derivative of the four-potential. Thus, we may expect that the four-potential is the derivative of a scalar function on spacetime, which we will call the pre-potential. Note that the usual differential of a gradient of a real-valued function is zero. Moreover, in order to describe rotational acceleration, the pre-potential must be complex valued. As a result, it is necessary to define a Lorentz-invariant conjugation on complexified spacetime. The four-potential is the conjugation of the gradient of the pre-potential. The electromagnetic field is then obtained by taking the curl of the four-potential. For a gravitational field, the pre-potential and the four-potential are derived in a similar fashion. However, getting from the four-potential to the field is handled differently.

The outline of the paper is as follows. In Section 2, we define our pre-potential and four-potential of any field, propagating with the speed of light, generated by a single source. The pre-potential $\psi(x)$ at $x$ is defined by the strength of the source and the relative position $r$ of the source at the retarded time. The relative position is a null vector in Minkowski spacetime. By introducing bipolar coordinates in spacetime, we associate to $r$ a complex angle $\zeta$ describing the relative direction of the source and the observer. The pre-potential $\psi(x)$ is defined everywhere outside the source as the product of the strength of the source and the direction angle $\zeta$. The direction angle of the source at the retarded time is easily calculated from the observed direction.

The next step is to find a representation $\pi_{L}$ of the Lorentz group under which the pre-potential is invariant. This representation is based on the identification of spacetime points with linear combinations of the Pauli matrices. We show that it is a spin-half representation and can be expressed by anti-self-dual matrices. Our approach here can also be cast in the language of the quaternions [9], the theory of spinors [10] and the Clifford or geometric algebra approaches to electrodynamics [11]. We show that the direction angle, and therefore, the pre-potential, are invariant under $\pi_{L}$.

We define a conjugation on complex Minkowski space which commutes with $\pi_{L}$ and then calculate the complex four-potential of the field. The four-potential is a complex extension of the Liénard-Wiechert potential [12]. The symmetries of the four-potential are investigated. It is surprising that field propagation with the speed of light is enough to obtain this four-potential. For example, we do not assume that the field satisfies an inverse-square law. We end Section 2 by showing that the pre-potential satisfies the wave equation.

In Section 3, we restrict the analysis to the electromagnetic field. We obtain the complex field tensor by taking the curl of the four-potential. The real part of the field tensor is the usual field tensor of a field generated by a single source. The complex part of the field tensor makes the complex tensor anti-self-dual. If one replaces the pre-potential with its complex conjugate, the resulting field tensor is self-dual. This shows that our description is independent of the choice of orientation.

Anti-self-duality is important for obtaining explicit formulas for charge evolution in electromagnetic fields $[13,14]$. The importance of self-duality with respect to the symmetry of Maxwell's equations is explained in [15]. In [16,17], a complex four-potential was used to obtain a dual-symmetric 
quantum field theory. However, the self-duality of the field derived from it was assumed. In [18] as well, two real potentials were used to study the quantum field theory of charged particles.

We end the paper by indicating how to extend the model to a field generated by many sources. We write the components of the field tensor in terms of the total pre-potential and the $\alpha$-matrices of Dirac. We obtain Maxwell's equations for the pre-potential.

\section{Pre-Potential and Four-Potential of a Field Generated by a Single Source}

In this section, we define the pre-potential of an arbitrary field propagating with the speed of light, generated by a single source. We will study its properties and derive the corresponding four-potential. All of the information about the field is contained in the pre-potential. We also study the Lorentz invariance and the symmetries of the pre-potential and the four-potential.

\subsection{Definition of the Pre-Potential of a Field Generated by a Single Source}

In this section, we construct the pre-potential of a field propagating with the speed of light, generated by a moving source. Let $M$ be four-dimensional Minkowski spacetime, with coordinates $x=x^{\mu}$, where $\mu=0,1,2,3$. The Minkowski inner product is

$$
x \cdot y=\eta_{\mu v} x^{\mu} y^{\nu}, \quad \eta=\operatorname{diag}(1,-1,-1,-1),
$$

for $x, y \in M$.

Consider the field generated by a single source, and denote by $\check{x}(\tau)=\breve{x}^{\mu}(\tau)$ the worldline of the source, parameterized by proper time parameter $\tau$. To define the pre-potential at the spacetime point $x=x^{\mu}$, let the point $\check{x}(\tau(x))$ be the unique point of intersection of the past light cone at $x$ with the worldline $\check{x}(\tau)$ of the source. The time $\tau(x)$ on the worldline of the source, corresponding to this intersection, is uniquely determined by the point $x$ and is called the retarded time. Since the field propagates with the speed of light, the field at $x$ is proportional to the strength of the source and may depend only on the source's relative position

$$
r(x)=x-\check{x}(\tau(x))
$$

at the retarded time $\tau(x)$. The relative position $r$ is a null vector with a positive time component. This vector belongs to the upper half-plane

$$
M_{+}=\left\{r \in M: \quad r^{0} \geq 0\right\}
$$

We stress that we do not assume that our field satisfies an inverse-square law. Nevertheless, for purposes of dimensional analysis, it is worthwhile to observe that the potential of a field obeying an inverse-square law is $U(r)=k / r$, for some constant $k$ defining the strength of the source. This potential has dimensions $\frac{[k]}{\text { length }}$. If this potential is to be the derivative of our pre-potential, our pre-potential should have dimensions $[k]$. Thus, the pre-potential should be the product of $k$ and a unit-free scalar describing the relative position null vector $r$. As we know, angles are unit-free scalars associated to vectors.

Introduce bipolar $(B P)$ coordinates $\left(\rho^{0}, \rho^{1}, \varphi, \theta\right)$ defined, for any $r \in M_{+}$, by

$$
r^{0}=\rho^{0} \cosh \theta, \quad r^{1}=\rho^{1} \cos \varphi, r^{2}=\rho^{1} \sin \varphi, r^{3}=\rho^{0} \sinh \theta .
$$

The angle $\varphi$ in these coordinates is the usual polar angle $\varphi$ in the plane $\Pi_{1}=\operatorname{Span}\left(r^{1}, r^{2}\right)$, equipped with the Euclidean metric, and is defined up to the addition of an integer multiple of $2 \pi$. To have $\varphi$ defined uniquely, we can restrict to the interval $0 \leq \varphi<2 \pi$, but this entails a loss of continuity. For the complementary plane $\Pi_{0}=\operatorname{Span}\left(r^{0}, r^{3}\right)$, the metric is hyperbolic, and thus the 
associated angle $\theta$ is hyperbolic. These coordinates lead to a simple form for the pre-potential, but to achieve this involves splitting $4 \mathrm{D}$ spacetime into a sum of two planes $\Pi_{0}$ and $\Pi_{1}$.

For any point $x \in M_{+}$, using (4), we define a local orthonormal frame $\left\{d_{\alpha}\right\}$ :

$$
\begin{aligned}
& d_{0}=\frac{\partial r}{\partial \rho^{0}}=(\cosh \theta, 0,0, \sinh \theta), d_{1}=\frac{\partial r}{\partial \rho^{1}}=(0, \cos \varphi, \sin \varphi, 0) \\
& d_{2}=\frac{1}{\rho^{1}} \frac{\partial r}{\partial \varphi}=(0,-\sin \varphi, \cos \varphi, 0), d_{3}=\frac{1}{\rho^{0}} \frac{\partial r}{\partial \theta}=(\sinh \theta, 0,0 \cosh \theta),
\end{aligned}
$$

and the dual basis $\left\{d^{\alpha}\right\}$ of co-vectors $d_{\mu}^{\alpha}=\eta_{\mu \nu} d_{\alpha}^{\nu}$. We use boldface indices for the BP basis and dual basis to distinguish from the usual basis. Note that differentiation by $\theta$ and $\varphi$ maps the local frame into itself:

$$
d_{0, \theta}=d_{3}, d_{3, \theta}=d_{0}, d_{1, \varphi}=d_{2}, d_{2, \varphi}=-d_{1} .
$$

In $B P$ coordinates, a null vector $r$ belongs to a hyperplane $\rho^{0}=\rho^{1}=\rho$ and is of the form

$$
r=\rho(\cosh \theta, \cos \varphi, \sin \varphi, \sinh \theta),
$$

or

$$
r=\rho\left(d_{0}+d_{1}\right) .
$$

The past light cone $L C_{x}$ about a point $x$ is

$$
L C_{x}=\{x-\rho(\cosh \theta, \cos \varphi, \sin \varphi, \sinh \theta): \rho>0,-\infty<\theta<\infty, 0 \leq \varphi<2 \pi\} .
$$

Note that if $r=0$, meaning that the point $x$ is on the worldline of the source, then $\theta$ and $\varphi$ are not defined.

As mentioned above, the pre-potential of the field is proportional to the strength $k$ of the source and depends on an angle determined by the relative position of the source. We propose the following definition.

Definition 1. For any $x \in M_{c}$ not on the worldline of the source, the pre-potential of the field generated by a single source of constant strength $k$ is defined by

$$
\psi(x)=k(\theta-i \varphi),
$$

a product of $k$ and the complex angle

$$
\zeta=\theta-i \varphi
$$

of its relative position $r(x)$ defined by (2).

Note that the field strength $k$ can be positive or negative.

We could have defined the angle to be $\zeta=\theta+i \varphi$. This would amount to a change of orientation of the spatial axes, and, later, would require using right multiplication in our representation of the Lorentz group instead of left multiplication. Since operators usually act from the left, we have chosen a minus sign in the complex angle. In Section 2.4, we will show that the pre-potential is invariant under a representation of the Lorentz group.

Note that any stellar observation can be translated into the complex angle. For any observation, we have the angles $\tilde{\varphi}, \tilde{\theta}$ of the spherical coordinates. We may assume $\varphi=\tilde{\varphi}$, and using (6), we obtain $\theta=\tanh ^{-1}(\cos \tilde{\theta})$. Thus, the complex angle $\zeta$ is calculated directly form the observation.

In order to restore the continuity lost by restricting to $0 \leq \varphi<2 \pi$, we may define the following continuous prepotential. 
Definition 2. For any $x \in M_{c}$ not on the worldline of the source, the "continuous pre-potential of the field generated by a single source" of constant strength $k$ is defined by

$$
\tilde{\psi}(x)=k e^{\zeta},
$$

where $\zeta$ is defined by (10).

As we show later, the four-potential derived from Definition 1 is a complex extension of the usual electromagnetic four-potential, while the continuous four-potential is closer to the quantum mechanics model.

We could have defined the pre-potential using the complex conjugate angle $\bar{\zeta}$. This is equivalent to a change of orientation.

The following properties of the derivatives of the complex angle with respect to $r^{\mu}$ will be needed later. Now, we must introduce two null-vectors $n=(1,0,0,-1)$ and $m=(0,-1,-i, 0)$. These are two of the four vectors of the Newman-Penrose tetrad [19]. Then $r \cdot n=\rho e^{\theta}$ and $r \cdot m=\rho e^{i \varphi}$, implying that $\zeta=\ln \frac{r \cdot n}{r \cdot m}=\ln (r \cdot n)-\ln (r \cdot m)$. Thus,

$$
\frac{\partial \zeta}{\partial r^{\mu}}=\frac{n_{\mu}}{r \cdot n}-\frac{m_{\mu}}{r \cdot m} .
$$

This implies that

$$
\nabla_{r} \zeta \cdot r=\frac{\partial \zeta}{\partial r^{\mu}} r^{\mu}=\frac{r^{\mu} n_{\mu}}{r \cdot n}-\frac{r^{\mu} m_{\mu}}{r \cdot m}=\frac{r \cdot n}{r \cdot n}-\frac{r \cdot m}{r \cdot m}=0,
$$

meaning that the gradient of $\zeta$ with respect to $r$ is perpendicular to $r$. Moreover, since $n, m$ are null, the d'Alembertian of $\zeta$ with respect to $r$ vanishes:

$$
\square_{r} \zeta=\eta^{\mu \nu} \frac{\partial^{2} \zeta}{\partial r^{\mu} \partial r^{v}}=-\frac{n \cdot n}{(r \cdot n)^{2}}+\frac{m \cdot m}{(r \cdot m)^{2}}=0 .
$$

\subsection{Matrix Representations of Spacetime}

Since the pre-potential is a complex-valued function on $M$, its gradient is a vector in complexified Minkowski space $M_{\mathcal{c}}$, which is $\mathbb{C}^{4}$ endowed with the unconjugated Minkowski inner product $x \cdot y=$ $\eta_{\mu v} x^{\mu} y^{\nu}$. In order to obtain a representation of the Lorentz group under which the pre-potential is invariant, we introduce the following identification of $M_{c}$ with $2 \times 2$ complex matrices:

$$
\Phi(x)=\left(\begin{array}{cc}
x^{0}+x^{3} & x^{1}+i x^{2} \\
x^{1}-i x^{2} & x^{0}-x^{3}
\end{array}\right)
$$

(see $[20,21]$ and references therein). The components of this matrix are the coordinates of $x$ with respect to the Newman--Penrose null tetrad, but identifying the vector as a matrix enables the use of additional mathematical tools such as matrix multiplication and determinants. We observe that matrix multiplication is associative but not commutative. Non-commutativity is one of the major distinctions between the classical and quantum models. Note that $(x)^{2}=\operatorname{det} \Phi(x)$.

Using the Pauli matrices

$$
\sigma_{0}=\left(\begin{array}{ll}
1 & 0 \\
0 & 1
\end{array}\right), \sigma_{1}=\left(\begin{array}{ll}
0 & 1 \\
1 & 0
\end{array}\right), \sigma_{2}=\left(\begin{array}{cc}
0 & i \\
-i & 0
\end{array}\right), \sigma_{3}=\left(\begin{array}{cc}
1 & 0 \\
0 & -1
\end{array}\right),
$$

we can write

$$
\Phi(x)=x^{\mu} \sigma_{\mu}
$$


The Pauli matrices satisfy the canonical anti-commutation relationships

$$
\left\{\sigma_{j}, \sigma_{k}\right\}=\frac{1}{2}\left(\sigma_{j} \sigma_{k}+\sigma_{k} \sigma_{j}\right)=\delta_{j, k} I \quad, \quad j, k=1,2,3,
$$

where $\delta_{j, k}$ is the Kronecker delta and $I$ is the identity. The multiplication rules for the Pauli matrices are

$$
\sigma_{0} \sigma_{j}=\sigma_{j} \sigma_{0}=\sigma_{j}, \sigma_{1} \sigma_{2}=-i \sigma_{3}, \sigma_{2} \sigma_{3}=-i \sigma_{1}, \sigma_{3} \sigma_{1}=-i \sigma_{2}
$$

The identification of $M_{\mathcal{c}}$ by the matrices $\Phi(x)$ is related to the quaternion approach [10], which has long been used in relativity, geometry and quantum mechanics. The quaternions have the form $q=q^{0}+q^{1} \mathbf{i}+q^{2} \mathbf{j}+q^{3} \mathbf{k}, q^{\mu} \in \mathbb{C}$. The multiplication rules (18) of the matrices $\sigma_{0}, i \sigma_{1}, i \sigma_{2}, i \sigma_{3}$ are the same as those of the quaternions $\{1, \mathbf{i}, \mathbf{j}, \mathbf{k}\}$. Note that the quaternion multiplication is associative but not commutative, as is matrix multiplication. This means that all of our results here can be translated into the language of the quaternions.

\subsection{Lorentz Group Representations of $M_{\mathcal{C}}$}

The Lorentz group is generated by boosts $B_{j}$ in the direction $x^{j}$ and rotation $R_{j}$ about the $x^{j}$ axis, for $j=1,2,3$. The regular representation $\pi$ of the Lorentz group on $M$ is by use of the Lorentz transformations defined by a velocity in the direction $x^{j}$ for boosts $B_{j}$, and multiplication by a rotation matrix about the $x^{j}$ axis for the rotation $R_{j}$. This representation has a natural extension to a representation on $M_{c}$.

Under the identification (14) of $M_{c}$ with $2 \times 2$ matrices, the representation $\pi$ may be obtained by multiplication of $\Phi(x)$ from both the left and the right by certain $2 \times 2$ matrices. This indicates that the regular representation $\pi$ can be decomposed into a product of two representations $\pi_{L}$ and $\pi_{R}$ of the Lorentz group on $M_{c}$, where $\pi_{L}$ acts by left multiplication on $\Phi(x)$ and $\pi_{R}$ acts by right multiplication on $\Phi(x)$.

To define a representation of the Lorentz group, it is enough to define the representation of the generators $\mathfrak{B}_{j}$ of boosts $B_{j}$ and the generators $\Re_{j}$ of rotations $R_{j}$.

Definition 3. Under the representation $\pi_{L}$, the generators $\mathfrak{B}_{j}$ of boosts in the direction of $x^{j}$ and the generators $\mathfrak{R}_{j}$ of rotation about the $x^{j}$ axis are represented as:

$$
\pi_{L} \mathfrak{B}_{j}(x)=\Phi^{-1}\left(\frac{1}{2} \sigma_{j} \Phi(x)\right), \pi_{L} \mathfrak{R}_{j}(x)=\Phi^{-1}\left(\frac{i}{2} \sigma_{j} \Phi(x)\right)
$$

for any $x \in M_{\mathcal{c}}$, where $\sigma_{j}$ are the Pauli matrices (15).

To show that $\pi_{L}$ is a representation of the Lorentz group, it is enough to check that $\pi_{L} \mathfrak{B}_{j}$ and $\pi_{L} \Re_{j}$ satisfy the same commutation relations as the corresponding generators of the Lorentz group. This follows directly from (17) and (18).

Under this representation, the boosts $B_{j}$ act by left multiplication of $\Phi(x)$ by $\exp \left(\frac{w}{2} \sigma_{j}\right)$ and rotations $R_{j}$ act by multiplication by $\exp \left(\frac{i \omega}{2} \sigma_{j}\right)$, for parameters $w, \omega$. From (17), it follows that

$$
\exp \left(\frac{w}{2} \sigma_{1}\right)=\left(\begin{array}{cc}
\cosh \frac{w}{2} & \sinh \frac{w}{2} \\
\sinh \frac{w}{2} & \cosh \frac{w}{2}
\end{array}\right), \exp \left(\frac{i \omega}{2} \sigma_{1}\right)=\left(\begin{array}{cc}
\cos \frac{\omega}{2} & i \sin \frac{\omega}{2} \\
i \sin \frac{\omega}{2} & \cos \frac{\omega}{2}
\end{array}\right)
$$


This implies that

$$
\pi_{L} B_{1}=\Phi^{-1}\left(\exp \left(\frac{w}{2} \sigma_{1}\right) \Phi(x)\right)=\left(\begin{array}{cccc}
\cosh \frac{w}{2} & \sinh \frac{w}{2} & 0 & 0 \\
\sinh \frac{w}{2} & \cosh \frac{w}{2} & 0 & 0 \\
0 & 0 & \cosh \frac{w}{2} & -i \sinh \frac{w}{2} \\
0 & 0 & i \sinh \frac{w}{2} & \cosh \frac{w}{2}
\end{array}\right)
$$

and

$$
\pi_{L} R_{1}=\Phi^{-1}\left(\exp \left(\frac{i w}{2} \sigma_{1}\right) \Phi(x)\right)=\left(\begin{array}{cccc}
\cos \frac{\omega}{2} & i \sin \frac{\omega}{2} & 0 & 0 \\
i \sin \frac{\omega}{2} & \cos \frac{\omega}{2} & 0 & 0 \\
0 & 0 & \cos \frac{\omega}{2} & \sin \frac{\omega}{2} \\
0 & 0 & -\sin \frac{\omega}{2} & \cos \frac{\omega}{2}
\end{array}\right) .
$$

The formulas for the boosts $B_{2}, B_{3}$ and rotations $R_{2}, R_{3}$ are similar. This establishes that $\pi_{L}$ is a spin-half representation of the Lorentz group.

Note that the generator $\mathfrak{B}_{j}$ of a boost in the direction of $x^{j}$ is associated with the acceleration in this direction, while the generator $\mathfrak{R}_{j}$ of rotation about the $x^{j}$ axis is associated with the corresponding rotational acceleration. Thus, as mentioned above, it is natural that the generator of a rotation is $i$ times the generator of a boost. Since the representation $\pi_{L}$ acts by left multiplication on the matrix $\Phi(x)$, it acts linearly on the columns of $\Phi(x)$. Hence, this representation has two invariant subspaces

$$
M_{1}=\left\{x: x^{1}+i x^{2}=0, x^{0}-x^{3}=0\right\} \quad, \quad M_{2}=\left\{x: x^{0}+x^{3}=0, x^{1}-i x^{2}=0\right\},
$$

corresponding to the first and second column, respectively.

The matrix representation of the generators (20) of boosts are the Majorana-Oppenheimer matrices (see [22])

$$
\left(K_{1}\right)_{\beta}^{\alpha}=\frac{1}{2}\left(\begin{array}{cccc}
0 & 1 & 0 & 0 \\
1 & 0 & 0 & 0 \\
0 & 0 & 0 & -i \\
0 & 0 & i & 0
\end{array}\right),\left(K_{2}\right)_{\beta}^{\alpha}=\frac{1}{2}\left(\begin{array}{cccc}
0 & 0 & 1 & 0 \\
0 & 0 & 0 & i \\
1 & 0 & 0 & 0 \\
0 & -i & 0 & 0
\end{array}\right),\left(K_{3}\right)_{\beta}^{\alpha}=\frac{1}{2}\left(\begin{array}{cccc}
0 & 0 & 0 & 1 \\
0 & 0 & -i & 0 \\
0 & i & 0 & 0 \\
1 & 0 & 0 & 0
\end{array}\right) .
$$

The generators of rotations are $i K_{j}, j=1,2,3$. (After lowering the indices, these become antisymmetric matrices).

We can define a dual representation $\pi_{R}$ by replacing the multiplication in (19) from the left by Pauli matrices $\sigma_{j}$ with multiplication by their complex conjugates $\bar{\sigma}_{j}$ from the right, and replacing $i$ with $-i$ for the generators of rotations. This representation can also be defined via the matrices $\bar{K}_{j}$, which are the complex conjugates of $K_{j}$. The matrices $\bar{K}_{j}$ are antisymmetric on $M_{c}$. The invariant subspaces of $\pi_{R}$ are the two subspaces corresponding to the rows of $\Phi(x)$. These representations commute, and the usual representation $\pi$ of the Lorentz group is the product of these representations: $\pi=\pi_{L} \pi_{R}$.

We now explain the meaning of the parameter $w$ in formula (20). A direct calculation shows that $\pi B_{1}=\pi_{L}\left(B_{1}\right) \pi_{R}\left(B_{1}\right)=\pi_{L}\left(B_{1}\right) \frac{g}{\pi_{L}\left(B_{1}\right)}$, which is the matrix of a Lorentz boost corresponding to the velocity $\mathbf{v}=(c \tanh w, 0,0)$. Thus, $w$ is the rapidity of $\mathbf{v}$, and $w / 2$ is the rapidity of the symmetric velocity of $v$ (see [23]). The operator $\pi_{L} B_{1}$ contains the usual boost in the $\left(x^{0}, x^{1}\right)$ plane, but with boost velocity equal to the symmetric velocity. An additional boost of the same magnitude acts on the 
$\left(x^{2}, i x^{3}\right)$ plane, which is the orthogonal complement of the $\left(x^{0}, x^{1}\right)$ plane. In the $\left(x^{2}, i x^{3}\right)$ plane, the Minkowski metric has the same signature as in the $\left(x^{0}, x^{1}\right)$ plane.

The meaning of the parameter $\omega$ in formula (21) is similar. A direct calculation shows that $\pi R_{1}=\pi_{L}\left(R_{1}\right) \pi_{R}\left(R_{1}\right)=\pi_{L}\left(R_{1}\right) \overline{\pi_{L}\left(R_{1}\right)}$, which is the matrix of a rotation by an angle $\omega$. The operator $\pi_{L} R_{1}$ contains a rotation in the $\left(x^{2}, x^{3}\right)$ plane by the half-angle $\omega / 2$. An additional rotation by $\omega / 2$ acts on the $\left(i x^{0}, x^{1}\right)$ plane, which is the orthogonal complement of the $\left(x^{2}, x^{3}\right)$ plane. In the $\left(i x^{0}, x^{1}\right)$ plane, the Minkowski metric has the same signature as in the $\left(x^{2}, x^{3}\right)$ plane.

The above considerations show that the representations $\pi_{L}$ and $\pi_{R}$ have symmetries not present in the usual representation $\pi$. For example, under $\pi$, a boost acts only on the plane spanned by the time direction and the direction of the boost, but leaves the orthogonal complement fixed. To describe the additional symmetry of $\pi_{L}$ and $\pi_{R}$, we define, as in [11], for any pair of vectors $a, b \in M_{c}$, an operator $a \wedge b$ by

$$
(a \wedge b) x=a(b \cdot x)-b(a \cdot x), \quad x \in M_{c} .
$$

For any basis $\left\{e_{\mu}\right\}$ of $M_{c}$, a natural basis of antisymmetric operators on $M_{c}$ is given by $e_{\mu v}=e_{\mu} \wedge e_{\nu}$. The Hodge dual operator $\star$ is a linear map defined by

$$
\star e_{\mu \nu}=\frac{1}{2} \epsilon^{\alpha \beta}{ }_{\mu \nu} e_{\alpha \beta}
$$

where $\epsilon$ is the Levi-Civita symbol. The square of this operator is minus the identity: $\star \star=-I$. To turn this operator into a symmetry, we define

$$
\Lambda=i \star .
$$

This is the helicity operator used in [24] . Obviously, $\Lambda^{2}=I$, implying that $\Lambda$ is a symmetry.

Definition 4. We will call an antisymmetric tensor $\mathcal{F}$ "self-dual" if

$$
\mathcal{F}=\Lambda \mathcal{F}
$$

and "anti-self-dual" if

$$
\mathcal{F}=-\Lambda \mathcal{F}
$$

For example, the Majorana-Oppenheimer matrices $K_{j}$ are anti-self-dual. Thus, the generators of the representation $\pi_{L}$ of Definition 3 are anti-self-dual. Similarly, the generators of the representation $\pi_{R}$ are self-dual. The representations $\pi_{L}$ and $\pi_{R}$ correspond to different helicities.

\subsection{Lorentz Invariance of the Pre-Potential and the Conjugation}

We now prove the following.

Claim 1. The pre-potential $\psi(x)$ defined by (9) and the continuous pre-potential $\tilde{\psi}$ defined by (11) are invariant under the representation $\pi_{L}$. The complex conjugates of $\psi(x)$ and $\tilde{\psi}(x)$ are invariant under the representation $\pi_{R}$. In addition, $\psi(x), \tilde{\psi}$ and their complex conjugates are invariant under scaling.

Using (6), $\Phi(r)$ becomes

$$
\Phi(r)=\rho\left(\begin{array}{cc}
e^{\theta} & e^{i \varphi} \\
e^{-i \varphi} & e^{-\theta}
\end{array}\right) .
$$

Since the determinant of $\Phi(r)$ is zero, the first row $R 1$ is proportional to the second row $R 2$, and the first column $C 1$ is proportional to the second column $C 2$. Explicitly,

$$
R 1=e^{\bar{\zeta}} R 2, \quad C 1=e^{\zeta} C 2,
$$

where $\zeta$ is defined by (10) and $\bar{\zeta}$ is its complex conjugate. 
Under the representation $\pi_{L}$, the boosts and rotations act by multiplication from the left of $\Phi(x)$ by $\exp \left(\sigma_{j} \omega / 2\right)$ and $\exp \left(i \sigma_{j} \omega / 2\right)$, respectively, for a parameter $\omega$. Since this operation is a linear map on the columns of $\Phi(x)$, the relation (29) between the columns is preserved. This implies that $e^{\zeta}$, and hence $\zeta$, are preserved under the representation $\pi_{L}$. Thus, both the continuous pre-potential $\tilde{\psi}(x)$ defined in Definition 2 and the pre-potential $\psi(x)$ defined by (9) are invariant under the representation $\pi_{L}$.

Similarly, the representation $\pi_{R}$, acting by multiplication from the right of $\Phi(x)$, preserves the relation between the rows, implying that $\bar{\zeta}$ is preserved under the representation $\pi_{R}$. Thus, the complex conjugate of the pre-potential and the continuous pre-potential are invariant under the representation $\pi_{R}$.

Since scaling is equivalent to multiplication of the matrix $\Phi(x)$ by the scale factor, scaling preserves the ratio (29) between the rows and the columns. Thus, the pre-potential, the continuous pre-potential and their conjugates remain the same after scaling. This proves the claim.

The complex electromagnetic field strength tensor $\mathcal{F}$ should be the derivative of a co-vector-valued four-potential $\mathcal{A}$, meaning that $\mathcal{F}=\nabla \wedge \mathcal{A}$. We cannot take $\mathcal{A}=\nabla \psi$, since then $\mathcal{F}=\nabla \wedge \nabla \psi=0$. Hence, we define a linear conjugation $\sharp$ on complexified spacetime $M_{c}$ and define the four-potential to be $\sharp \nabla \psi$.

We define the conjugation $\sharp: M_{\mathcal{c}} \rightarrow M_{\mathcal{c}}$ by

$$
\sharp x=\Phi^{-1}\left(\Phi(x) \sigma_{3}\right) .
$$

Clearly, $\sharp^{2}=I$, and the matrix $\Phi(\sharp x)$ differs from the matrix $\Phi(x)$ only by a change of sign of the second column.

For the regular representation $\pi$ of the Lorentz group, there are two invariant subspaces, $\Re M_{c}$ and $\Im M_{c}$. Complex conjugation changes the sign of the second invariant subspace. Similarly, for the representation $\pi_{L}$, there are two invariant subspaces, $M_{1}$ and $M_{2}$, defined by (22), and the conjugation $\sharp x$ changes the sign of the second invariant subspace.

Claim 2. The conjugation $\sharp$ commutes with the representation $\pi_{L}$ of the Lorentz group.

Since matrix multiplication is associative, and $\sharp$ acts by multiplication of $\Phi(x)$ from the right, while the representation $\pi_{L}$ acts by multiplication of $\Phi(x)$ from the left, the conjugation $\sharp$ commutes with the action of $\pi_{L}$. This proves the claim.

Similarly, a conjugation of $x$ which changes the sign of the second row of $\Phi(x)$ is invariant under the representation $\pi_{R}$.

Under the Pauli matrix representation, the $B P$ frame is

$$
\begin{gathered}
\Phi\left(d_{\mathbf{0}}\right)=\left(\begin{array}{cc}
e^{\theta} & 0 \\
0 & e^{-\theta}
\end{array}\right), \Phi\left(d_{\mathbf{1}}\right)=\left(\begin{array}{cc}
0 & e^{i \varphi} \\
e^{-i \varphi} & 0
\end{array}\right), \\
\Phi\left(d_{2}\right)=\left(\begin{array}{cc}
0 & i e^{i \varphi} \\
-i e^{-i \varphi} & 0
\end{array}\right), \Phi\left(d_{3}\right)=\left(\begin{array}{cc}
e^{\theta} & 0 \\
0 & -e^{-\theta}
\end{array}\right) .
\end{gathered}
$$

Thus, the conjugation on this frame is

$$
\sharp d_{0}=d_{3}, \sharp d_{3}=d_{0}, \sharp d_{1}=i d_{2}, \sharp d_{2}=-i d_{1} .
$$

On the local frame of co-vectors, defined by $d_{\mu}^{\alpha}=\eta_{\mu \nu} d_{\alpha^{\prime}}^{v} \alpha=0,1,2,3$, the conjugation is

$$
\sharp d^{0}=-d^{3}, \sharp d^{3}=-d^{0}, \sharp d^{1}=i d^{2}, \sharp d^{2}=-i d^{1} .
$$

\subsection{The Four-Potential of a Moving Source}

We now define the complex four-potential of a moving source. 
Definition 5. The complex four-potential $\mathcal{A}$ is defined by

$$
\mathcal{A}=\sharp \nabla \psi,
$$

where $\psi$ is defined by Definition 1 and $\sharp$ is defined by Equation (30).

To derive an explicit formula for the complex four-potential of a moving source, we first compute the derivatives of the relative position $r(x)$ defined by (2). The partial derivative $r_{, \mu}=\partial r / \partial x^{\mu}$ is

$$
r_{, \mu}^{\alpha}=x_{, \mu}^{\alpha}-\check{x}_{, \mu}^{\alpha}(\tau(x))=x_{, \mu}^{\alpha}-\partial \check{x}^{\alpha} / \partial \tau \tau_{, \mu}=x_{, \mu}^{\alpha}-u^{\alpha} \tau_{, \mu}
$$

where $u$ denotes the four-velocity of the source at the retarded time. Note that $x_{, \mu}^{\alpha}=\delta_{\mu}^{\alpha}$. The vector $r(x)$ is null, implying that $r \cdot r_{, \mu}=0$. Thus, using the above and $\eta_{\alpha \beta} \delta_{\mu}^{\beta}=\eta_{\alpha \mu}$, we get

$$
0=r \cdot r_{, \mu}=\eta_{\alpha \beta} r^{\alpha} r_{, \mu}^{\beta}=\eta_{\alpha \beta} r^{\alpha}\left(\delta_{\mu}^{\beta}-u^{\beta} \tau_{, \mu}\right)=\eta_{\alpha \mu} r^{\alpha}-(r \cdot u) \tau_{, \mu}=r_{\mu}-(r \cdot u) \tau_{, \mu}
$$

Hence, the derivative of the retarded time is

$$
\tau(x)_{, \mu}=\frac{r_{\mu}}{r \cdot u},
$$

and hence,

$$
r_{, \mu}^{\alpha}=\delta_{\mu}^{\alpha}-\frac{u^{\alpha} r_{\mu}}{r \cdot u}
$$

Since $(r)^{2}=0$ and $(u)^{2}=1$, the inner product $r \cdot u$ is always non-zero, so equations (34) and (35) are always well defined.

Equations (7) and (5) yield

$$
r_{, \mu}=\rho_{, \mu} d_{0}+\rho_{, \mu} d_{1}+\rho \varphi_{, \mu} d_{2}+\rho \theta_{, \mu} d_{3} .
$$

Taking the dot product of this equation with $d_{0}$ and using (35) and (7) yields

$$
\rho_{, \mu}=d_{\mathbf{0}} \cdot\left(x_{, \mu}-\frac{u r_{\mu}}{r \cdot u}\right)=\frac{d_{\mu}^{0} \rho\left(\left(d_{0}+d_{\mathbf{1}}\right) \cdot u\right)-\rho\left(d_{\mu}^{0}-d_{\mu}^{\mathbf{1}}\right)\left(d_{\mathbf{0}} \cdot u\right)}{r \cdot u} .
$$

Using (23) and denoting $d_{01}=d_{0} \wedge d_{1}$, this can be rewritten as

$$
\frac{\nabla \rho}{\rho}=\eta \frac{d_{01} u}{r \cdot u}
$$

Taking now, the dot product of Equation (36) with $d_{3}$ and $d_{2}$, respectively, and using (35) and (7), we get

$$
\rho \theta_{, \mu}=d_{3} \cdot\left(\frac{u r_{\mu}}{r \cdot u}-x_{, \mu}\right)=\frac{\rho\left(d_{\mu}^{0}+d_{\mu}^{1}\right)\left(d_{\mathbf{3}} \cdot u\right)-d_{\mu}^{3} \rho\left(\left(d_{0}+d_{1}\right) \cdot u\right)}{r \cdot u},
$$

or

$$
\rho \nabla \theta=\eta \rho \frac{\left(\left(d_{0}+d_{1}\right) \wedge d_{3}\right) u}{r \cdot u}=\eta \frac{\left(r \wedge d_{3}\right) u}{r \cdot u},
$$

and

$$
\rho \varphi_{, \mu}=d_{\mathbf{2}} \cdot\left(\frac{u r_{\mu}}{r \cdot u}-x_{, \mu}\right)=\frac{\rho\left(d_{\mu}^{0}+d_{\mu}^{\mathbf{1}}\right)\left(d_{\mathbf{2}} \cdot u\right)-d_{\mu}^{2} \rho\left(\left(d_{\mathbf{0}}+d_{\mathbf{1}}\right) \cdot u\right)}{r \cdot u}
$$

or

$$
\rho \nabla \varphi=\eta \rho \frac{\left(\left(d_{0}+d_{1}\right) \wedge d_{2}\right) u}{r \cdot u}=\eta \frac{\left(r \wedge d_{2}\right) u}{r \cdot u} .
$$


Thus, the gradient of the pre-potential defined by (9) is

$$
\nabla \psi=k(\nabla \theta-i \nabla \varphi)=k \eta \frac{\left.\left(d_{0}+d_{\mathbf{1}}\right) \wedge\left(d_{\mathbf{3}}-i d_{\mathbf{2}}\right)\right) u}{r \cdot u} .
$$

Using Equation (32), the complex four-potential (Equation (33)) is

$$
\mathcal{A}=\sharp \nabla \psi=k \eta \frac{\left(d_{\mathbf{0}}-d_{\mathbf{1}}\right)\left(\left(d_{\mathbf{0}}+d_{\mathbf{1}}\right) \cdot u\right)-\left(\left(d_{\mathbf{3}}-i d_{\mathbf{2}}\right) \cdot u\right)\left(d_{\mathbf{3}}+i d_{\mathbf{2}}\right)}{r \cdot u} .
$$

The numerator is a sum of three components. The first term is

$$
d_{0}\left(d_{0} \cdot u\right)-d_{1}\left(d_{1} \cdot u\right)-d_{2}\left(d_{2} \cdot u\right)-d_{3}\left(d_{3} \cdot u\right)=u,
$$

since this is the decomposition of $u$ by the frame. The second term is

$$
d_{0}\left(d_{1} \cdot u\right)-d_{1}\left(d_{0} \cdot u\right)=d_{01} u,
$$

which, by Equation (38), can be identified as the numerator of $\rho^{-1} \nabla \rho$. The last term, the only imaginary one, is

$$
i d_{3}\left(d_{2} \cdot u\right)-i d_{2}\left(d_{3} \cdot u\right)=-i d_{23} u \text {. }
$$

Thus,

$$
\mathcal{A}=k \frac{\eta u}{r \cdot u}+k \nabla \ln \rho-i k \eta \frac{d_{23} u}{r \cdot u} .
$$

The first term is the Liénard-Wiechert potential of the electromagnetic field of a moving source. The second term is a gauge, since it is the gradient of a scalar function. The last term is purely imaginary. Thus, the real part of $\mathcal{A}$ is a four-potential which properly defines the electromagnetic field of a moving source. As we show later, the complex part is needed to make the field strength anti-self-dual.

If we use the continuous pre-potential (Equation (11)), then the continuous complex four-potential is

$$
\tilde{\mathcal{A}}=\sharp \nabla \tilde{\psi}=\mathcal{A} \tilde{\psi} .
$$

In the Dirac equation, the four-potential of the external field is an operator acting on the wave function [25]. Thus, the continuous four-potential is closer to the quantum mechanics model.

If we change orientation and define the pre-potential to be $\bar{\psi}(x)$, then in Definition 5, we use the conjugation $\sharp$, which is invariant under $\pi_{R}$. The resulting four-potential is then the complex conjugate of the four-potential (42).

\subsection{The Symmetry of the Complex Four-Potential}

Let

$$
A=k \frac{\eta u}{r \cdot u}
$$

be the Liénard-Wiechert potential. From (41), we can write the complex four potential $\mathcal{A}$ as

$$
\mathcal{A}=\frac{k \eta}{r \cdot u}\left(u+\left(d_{01}-i d_{23}\right) u\right)=A+\eta S \eta A
$$

where $S$ is the operator

$$
S=d_{01}-i d_{23} .
$$

Using the definition of $d_{01}$ and $d_{23}$, it is easily verified that $S$ is an instance of symmetry. 
Claim 3. Using the Pauli matrix representation, the operator $S=d_{01}-i d_{23}$ acts on $x \in M_{\mathcal{C}}$ by

$$
\Phi(S x)=-\Phi(x)\left(\begin{array}{cc}
0 & e^{-\zeta} \\
e^{\zeta} & 0
\end{array}\right)
$$

for $\Phi$ defined by (14) and the complex angle $\zeta$ defined by (10). Moreover, $S$ commutes with the representation $\pi_{L}$.

It is straightforward to verify (47) by checking the action of $S$ on the $B P$ basis. Note that $\zeta$ is Lorentz invariant under the representation $\pi_{L}$. Since this representation acts by left multiplication on $\Phi(x)$, while $S$ acts by right multiplication, the operator $S$ and the operators of $\pi_{L}$ commute. This proves the Claim.

The eigenvectors of $S$ corresponding to the eigenvalues \pm 1 are spacetime points $x$ for which the columns of $\Phi(x)$ satisfy $C 1=\mp e^{\zeta} C 2$, respectively. For example, using (29), the relative position $r$ of the source at the retarded time with respect to the observer is mapped by $S$ to $-r$, which is the relative position of the observer with respect to the source at the retarded time. Since $\Phi(\sharp r)$ is obtained from $\Phi(r)$ by changing the sign of the second column, we get $S(\sharp r)=\sharp r$.

The operator $P=\frac{1}{2}(1+S)$ is a projection on $M_{\mathcal{C}}$ which commutes with $\pi_{L}$ and satisfies $\operatorname{Pr}=$ $0, P(\sharp r)=\sharp r$. Using (47), for any $x \in M_{\mathcal{C}}$, the norm of $P(x)$ is

$$
\operatorname{det} \Phi(P(x))=\frac{1}{4} \operatorname{det} \Phi(x) \operatorname{det}\left(\begin{array}{cc}
1 & -e^{-\zeta} \\
-e^{\zeta} & 1
\end{array}\right)=0
$$

Note that from the definition of $S$, it follows that $S u \cdot u=0$. This implies that $S u$ in purely spatial in the frame co-moving to the source. We have thus proven the following claim.

Claim 4. The complex four potential $\mathcal{A}$, defined by (45), is a scalar multiple of the null vector $u+S u$. In the frame co-moving to the source, the norms of the time and space components of $u+S u$ are 1 and -1 , respectively.

Moreover, $\mathcal{A}$ is twice the projection $P$ of the Liénard-Wiechert potential $A$ of the field of a single source.

\subsection{The Pre-Potential and the Wave Equation}

We show that a single-source pre-potential $\psi(x)$ satisfies the wave equation. To prove this, we will need the following formulas: Using (34),

$$
u_{, \mu}=u_{, \tau} \tau_{, \mu}=a \tau_{, \mu}=\frac{a r_{\mu}}{r \cdot u}
$$

where $a$ is the acceleration of the source at the retarded time. Using (35), (48) and $(u)^{2}=1$ yields

$$
(r \cdot u)_{, \mu}=r_{, \mu}^{\alpha} u_{\alpha}+u_{, \mu}^{\alpha} r_{\alpha}=u_{\mu}-\frac{r_{\mu}}{r \cdot u}+\frac{(r \cdot a) r_{\mu}}{r \cdot u}
$$

and

$$
\nabla \frac{1}{r \cdot u}=\eta \frac{-u}{(r \cdot u)^{2}}+\eta \frac{r-(r \cdot a) r}{(r \cdot u)^{3}}
$$

Claim 5. The single-source pre-potential $\psi(x)$, defined by (9), satisfies the wave equation $\square \psi(x)=0$ for any $x$ outside the source.

Since $\psi(x)$ is proportional to the complex angle $\zeta(x)=\theta-i \varphi$, it is enough to show that $\square \zeta(x)=0$. We get

$$
\square \zeta(x)=\eta^{\mu \nu} \frac{\partial}{\partial x^{\nu}}\left(\frac{\partial \zeta}{\partial r^{\kappa}} \frac{\partial r^{\kappa}}{\partial x^{\mu}}\right)=\eta^{\mu \nu} \frac{\partial^{2} \zeta}{\partial r^{\kappa} \partial r^{\alpha}} \frac{\partial r^{\kappa}}{\partial x^{\mu}} \frac{\partial r^{\alpha}}{\partial x^{\nu}}+\eta^{\mu \nu} \frac{\partial \zeta}{\partial r^{\kappa}} \frac{\partial^{2} r^{\kappa}}{\partial x^{\mu} \partial x^{\nu}}
$$


From (35), using that $r$ is null,

$$
\eta^{\mu \nu} \frac{\partial r^{\kappa}}{\partial x^{\mu}} \frac{\partial r^{\alpha}}{\partial x^{\nu}}=\eta^{\kappa \alpha}-\frac{u^{\kappa} r^{\alpha}+u^{\alpha} r^{\kappa}}{r \cdot u}
$$

and from (13) and using the symmetry of mixed differentiation, the first term in (50) is

$$
\eta^{\mu \nu} \frac{\partial^{2} \zeta}{\partial r^{\kappa} \partial r^{\alpha}} \frac{\partial r^{\kappa}}{\partial x^{\mu}} \frac{\partial r^{\alpha}}{\partial x^{v}}=\eta^{\kappa \alpha} \frac{\partial^{2} \zeta}{\partial r^{\kappa} \partial r^{\alpha}}-2 \frac{\partial^{2} \zeta}{\partial r^{\kappa} \partial r^{\alpha}} \frac{u^{\kappa} r^{\alpha}}{r \cdot u}=-2 \frac{u^{\kappa} r^{\alpha}}{r \cdot u} \frac{\partial^{2} \zeta}{\partial r^{\kappa} \partial r^{\alpha}} .
$$

Using (35) once more, we obtain

$$
\eta^{\mu \nu} \frac{\partial^{2} r^{\kappa}}{\partial x^{\mu} \partial x^{v}}=\eta^{\mu \nu} \frac{\partial}{\partial x^{v}} \frac{\partial}{\partial x^{v}} \frac{\partial r^{\kappa}}{\partial x^{\mu}}=\eta^{\mu \nu} \frac{\partial}{\partial x^{v}}\left(\delta_{\mu}^{\kappa}-\frac{u^{\kappa} r_{\mu}}{r \cdot u}\right)=-\eta^{\mu \nu} \frac{\partial}{\partial x^{v}} \frac{u^{\kappa} r_{\mu}}{r \cdot u}
$$

Combining (48) and (49) yields

$$
\frac{\partial}{\partial x^{v}} \frac{u^{\kappa}}{r \cdot u}=\frac{a^{\kappa} r_{v}}{(r \cdot u)^{2}}-\frac{u^{\kappa} u_{v}}{(r \cdot u)^{2}}+\frac{u^{\kappa} r_{v}(1-(r \cdot a))}{(r \cdot u)^{3}} .
$$

Since $r^{v} r_{v}=0$, we obtain

$$
\eta^{\mu \nu} \frac{\partial^{2} r^{\kappa}}{\partial x^{\mu} \partial x^{v}}=-\frac{\partial}{\partial x^{v}}\left(r^{v} \frac{u^{\kappa}}{r \cdot u}\right)=-\left(\delta_{v}^{v}-\frac{u^{v} r_{v}}{r \cdot u}\right) \frac{u^{\kappa}}{r \cdot u}+r^{v} \frac{u^{\kappa} u_{v}}{(r \cdot u)^{2}}=(-3+1) \frac{u^{\kappa}}{r \cdot u}=-2 \frac{u^{\kappa}}{r \cdot u} .
$$

Finally, using (12), we arrive at

$$
\square \zeta(x)=-2 \frac{u^{\kappa}}{r \cdot u}\left(\frac{\partial^{2} \zeta}{\partial r^{\kappa} \partial r^{\alpha}} r^{\alpha}+\frac{\partial \zeta}{\partial r^{\kappa}}\right)=-2 \frac{u^{\kappa}}{r \cdot u} \frac{\partial}{\partial r^{\kappa}}\left(\frac{\partial \zeta}{\partial r^{\alpha}} r^{\alpha}\right)=0
$$

This proves the claim.

\section{The Electromagnetic Field Tensor of a Moving Source and Its Self-Duality}

To this point, our results are valid for any field propagating with the speed of light. However, to obtain the evolution equation, we proceed differently for gravitational and electric fields. For a gravitational field, we use the four-potential to obtain a metric on spacetime and describe motion by the geodesic equation with respect to this metric. From this point on, we will consider only electromagnetic fields.

We derive first the tensor $F$ corresponding to the Liénard-Wiechert potential $A=k \frac{\eta u}{r \cdot u}$. For a covector $w$, define the wedge product $\nabla \wedge w$ by

$$
(\nabla \wedge w)_{\alpha \beta}=w_{\alpha, \beta}-w_{\beta, \alpha}
$$

Then, Equation (48) implies that

$$
\nabla \wedge \eta u=\eta \frac{r \wedge a}{r \cdot u}
$$

Using (48) and (49) yields

$$
\begin{aligned}
F_{\mu v}=\nabla & \wedge A=k\left(\nabla \wedge \frac{\eta u}{r \cdot u}\right)=k \frac{\nabla \wedge \eta u}{r \cdot u}+k \nabla \frac{1}{r \cdot u} \wedge \eta u \\
& =k \eta\left(\frac{r \wedge a}{(r \cdot u)^{2}}+\frac{r \wedge u-(r \cdot a) r \wedge u}{(r \cdot u)^{3}}\right),
\end{aligned}
$$


and the mixed electromagnetic field tensor is

$$
F=F^{\mu}{ }_{v}=k\left(\frac{r \wedge u}{(r \cdot u)^{3}}+\frac{r \wedge((a \wedge u) r)}{(r \cdot u)^{3}}\right) .
$$

The first term is the near field, which falls off as $1 / r^{2}$. The second term is the radiation field, which exists only when the source is accelerating, and it falls off as $1 / r$.

The complex electromagnetic field tensor $\mathcal{F}$ can be defined from the four-potential $\mathcal{A}$ via $\mathcal{F}=$ $\nabla \wedge \mathcal{A}$ or $\mathcal{F}_{\alpha \beta}=\mathcal{A}_{\alpha, \beta}-\mathcal{A}_{\beta, \alpha}$. We show that first.

Claim 6. The tensor $\mathcal{F}(x)=\nabla \wedge(\sharp \psi(x))$, with $\sharp$ defined by Equation (30), is anti-self-dual.

Since the matrix form of the gradient is $\nabla \psi=\psi_{, \mu} \sigma^{\mu}$, the matrix form of the four-potential is $\mathcal{A}=\sharp \nabla \psi=\psi_{, \mu} \sigma^{\mu} \sigma^{3}$, or, using Equation (18),

$$
\mathcal{A}=\left(\psi_{, 3}, i \psi, 2,-i \psi, 1, \psi_{, 0}\right)
$$

From this, it follows that

$$
\mathcal{F}_{01}=\mathcal{A}_{0,1}-\mathcal{A}_{1,0}=\psi_{, 31}-i \psi_{, 02}, \quad \mathcal{F}_{23}=\mathcal{A}_{2,3}-\mathcal{A}_{3,2}=-i \psi_{, 13}-\psi_{, 20},
$$

which implies $\mathcal{F}_{23}=-i \mathcal{F}_{01}$. Similarly,

$$
\mathcal{F}_{02}=\mathcal{A}_{0,2}-\mathcal{A}_{2,0}=\psi_{, 32}+i \psi_{, 10}, \quad \mathcal{F}_{31}=\mathcal{A}_{3,1}-\mathcal{A}_{1,3}=\psi_{, 01}-i \psi_{, 23},
$$

which implies $\mathcal{F}_{31}=-i \mathcal{F}_{02}$. But

$$
\mathcal{F}_{03}=\mathcal{A}_{0,3}-\mathcal{A}_{3,0}=\psi_{, 33}-\psi_{, 00}, \quad \mathcal{F}_{12}=\mathcal{A}_{1,2}-\mathcal{A}_{2,1}=i \psi_{, 22}+i \psi_{, 11} .
$$

This implies that $\mathcal{F}_{12}=-i \mathcal{F}_{03}$ if and only if

$$
\square \psi(x)=\psi_{, 00}-\psi_{, 11}-\psi_{, 22}-\psi_{, 33}=0 .
$$

By Claim (5), the previous equation holds. Therefore, by the definition (26) of an anti-self-dual operator, the tensor $\mathcal{F}(x)$ is anti-self-dual. This proves the claim.

By Claim 6, the tensor $\mathcal{F}$ is anti-self-dual and can be written as

$$
\mathcal{F}=F-i \star F,
$$

where $F=\nabla \wedge A$ is the real electromagnetic field tensor and $A=\Re \mathcal{A}=k \frac{\eta u}{r \cdot u}$. For the orientation corresponding to $\bar{\psi}(x)$, the corresponding complex tensor $\mathcal{F}$ is self-dual.

Any electromagnetic field is a sum or integral of the fields generated by its sources. Thus, the pre-potential is also an integral of the pre-potentials of the sources. For any point $x \in M$, the pre-potential will be defined by the distribution and the position of the source charges on the backward light cone $L C_{x}$ about this point. Using bipolar coordinates (4), any point in $L C_{x}$ is of the form $x-r$, with the null vector $r$ defined by (6). Thus, any point in $L C_{x}$ is defined by $(\rho, \theta, \varphi)$, with $0 \leq \rho<\infty,-\infty<\theta<\infty, 0 \leq \varphi<2 \pi$. The spatial volume element is $d v=\rho^{2} d \rho \cosh \theta d \theta d \varphi$.

Denote by $q(\check{x})$ the source charge density at $\check{x}$. The pre-potential at $x$ is then

$$
\begin{gathered}
\psi(x)=\int_{L C_{x}}(\theta-i \varphi) q(x-r) d v= \\
=\int_{0}^{\infty} \rho^{2} d \rho \int_{-\infty}^{\infty} \cosh \theta \int_{0}^{2 \pi}(\theta-i \varphi) q(x-r) d \varphi d \theta .
\end{gathered}
$$


We define matrices $\alpha_{j}$ for $j=1,2,3$ on $M_{c}$ by

$$
\Phi\left(\alpha_{j}(x)\right)=\sigma_{j} \Phi(x) \sigma_{3}
$$

These matrices are the $\alpha$-matrices of Dirac. It can be shown [7] that the Faraday vector $\mathbf{F}=\mathbf{E}+i \mathbf{B}$ describing the electromagnetic field can be calculated from the pre-potential by

$$
F_{j}(x)=\alpha_{j}(x)^{\mu v} \psi, \mu \nu .
$$

The Maxwell equations for the pre-potential become

$$
\sharp \nabla(\square \psi(x))=J(x),
$$

where $J(x)$ is the four-current density at $x$.

\section{Discussion}

The search for a complete, alternative formulation of classical electrodynamics in terms of a single scalar function has a long history; see $[1,4,26]$ and references therein. In this paper, we have constructed a pre-potential that is a single complex-valued function on spacetime, and we have shown that it contains all the information of the field. Our pre-potential is valid for any field which propagates with the speed of light; in particular, electromagnetic and gravitational fields.

We considered first, a field generated by a single source and then extended our results to any number of sources. By using bipolar coordinates (4), we introduced a complex angle $\zeta$ to describe the relative position of the observer and the source of the field at the retarded time. The pre-potential $\psi(x)$ is defined for any spacetime point $x$ outside the sources. At the observation point $x$, the pre-potential $\psi(x)$, defined in Definition 1, is the product of the strength of the source and $\zeta$. However, $\psi(x)$ has jump discontinuities which can be removed by redefining the pre-potential, as in Definition 2. Both pre-potentials are defined only from the strength of the source and the observed angular direction of the source at the retarded time, without the need to know the distance to the source or the source's velocity and acceleration. Note that the electromagnetic tensor $F_{\mu \nu}$ of the field of a moving charge depends on the strength, the direction angle, the distance, velocity and acceleration of the source at the retarded time; the four-potential $A_{\mu}$ depends on all of the above, except the acceleration, while $\psi(x)$ depends only on the strength of the source and the observed angle. The effect of a change of orientation in our model is expressed by taking the complex conjugate $\bar{\psi}(x)$ as the pre-potential.

To define the symmetries of the pre-potential, we use the spacetime identification (14) $\Phi(x)=x^{\mu} \sigma_{\mu}$, where $\sigma_{\mu}$ are the Pauli matrices. This already enables the use of matrix multiplication, which is not commutative. The generators of the regular representation $\pi$ of the Lorentz group act by multiplication of these matrices from left and right by multiples of Pauli matrices. This representation can be decomposed as a product of two representations $\pi_{L}$, acting from the left, and its complex adjoint $\pi_{R}$, acting from the right. Both representations are spin-half representations. We have shown that the pre-potential $\psi(x)$ is Lorentz invariant under $\pi_{L}$, and that $\bar{\psi}(x)$ is Lorentz invariant under $\pi_{R}$. The pre-potential satisfies the wave equation (Claim 5).

The helicity operator defines an important symmetry on the anti-symmetric tensors. The representation $\pi_{L}$ acts by anti-self-dual operators, while the representation $\pi_{R}$ acts by self-dual operators on complexified spacetime.

To define the four-potential of the field from the pre-potential, we defined an adjoint operator on spacetime. We recognized that the representation $\pi_{L}$ has two invariant subspaces, corresponding to the columns of $\Phi(x)$. The representation $\pi_{R}$ also has two invariant subspaces, corresponding to the rows of $\Phi(x)$. This observation helps to define a conjugation by changing the sign of one of the invariant subspaces. This conjugation is Lorentz invariant under the corresponding representation. 
The four-potential $\mathcal{A}$ is defined (33) as the conjugate of the gradient of $\psi(x)$. Taking advantage of the natural basis associated with bipolar coordinates, we calculated the four-potential of a field of a moving source and found (Equation (42)) that it is a complex extension of the known Liénard-Wiechert potential. It is surprising that the Liénard-Wiechert potential can be derived without assuming any specific law of the field, only assuming that it propagates with the speed of light. For example, we do not assume that the field satisfies an inverse-square law.

In Claim 4, we showed that our complex four-potential (45) is a null vector. In the frame co-moving to the source, the time component is the usual scalar potential. The vector part has the same (in absolute value) norm as the time component and depends on the relative position of the observer and the source at the retarded time. We obtained a Lorentz invariant formula (47) for this vector part.

There are two ways to derive the field strength from the four-potential. The first one, used here, assumes that the field strength is the derivative of this potential, as it is assumed for the electromagnetic field. The second way is to define a metric based on the four-potential and to assume that the evolution is by a geodesic with respect this metric, as in the general relativity approach to the gravitational field. For the electromagnetic field, the field strength tensor is proportional to the source strength $k$, which can be positive or negative, reflecting that the force generated by the field can be attractive or repulsive. On the other hand, for gravitational fields, when we construct the metric from the four-potential, the field strength will be proportional to $k^{2}$. This coincides with the fact that gravitational fields are always attractive.

We derived the electromagnetic field strength tensor from the four-potential. We have shown in Claim 6 that a field tensor derived by use of a pre-potential is anti-self-dual. The complex four-potential derived from $\bar{\psi}(x)$, using the conjugation associated with $\pi_{R}$, is the complex conjugate of the four-potential associated with $\psi(x)$ and leads to a self-dual electromagnetic field tensor.

It is known that Maxwell's equations are invariant under the conformal group. We have shown here that our pre-potential is invariant under the Poincare group and scaling. To obtain invariance under the full conformal group, one need only check invariance with respect to the special conformal transformation (also called the acceleration transformation) [27]. Since this transformation is not linear, it maps the linear interval connecting the source at the retarded time and the observer to part of a null geodesic, which may not be linear. Nevertheless, at the spacetime point $x$, one may define the tangent vector $\check{r}$ to this null geodesic, which is a null vector. The null vector $\breve{r}$ plays the role of the relative position $r$. One can define $\breve{\theta}$ and $\breve{\varphi}$ for this null vector with respect to a local tetrad. Based on the invariance of the complex angle $\zeta$ under $\pi_{L}$, we expect that this complex angle will be the same as before the special transformation. A rigorous proof is still needed.

Assuming that the field from several sources is the sum or integral of the fields of each of the sources, we presented a formula (54) for the pre-potential of such a field. The Faraday vector of the electromagnetic field can be derived from the pre-potential by contracting the second derivatives of the pre-potential with the $\alpha$-matrices of Dirac. Maxwell's equations connect the pre-potential to the sources of the field via the d'Alembertian of the pre-potential, followed by its conjugate gradient.

The representation of a field propagating with the speed of light by the pre-potential is a model applicable both for gravitational and electromagnetic fields. The model presented here shares some characteristics with quantum mechanics, such as complex-valued functions, non-commuting products, spin-half representations, and the $\alpha$-matrices of Dirac. Therefore, we hope that this model will be an interface between classical and quantum physics.

Parts of this paper were done as part of final undergraduate projects in the Applied Physics Department of the Jerusalem College of Technology by students S. Gwertzman and D. H. Gootvilig, under the supervision of the first author.

Author Contributions: conceptualization, Y.F.; methodology, Y.F. and D.H.G.; software, T.S.; validation, Y.F. and T.S.; formal analysis, Y.F. and D.H.G.; writing—original draft preparation, Y.F. and T.S.; writing—review and editing, Y.F. and T.S.; supervision, Y.F.; project administration, Y.F.

Funding: This research received no external funding. 
Acknowledgments: The authors would like to thank the referees for helpful comments and suggestions. The first author would like to thank Ivan Fernandez-Corbaton for pointing out the importance of self-duality.

Conflicts of Interest: The authors declare no conflicts of interest.

\section{References}

1. Whittaker, E.T. On An Expression of the electromagnetic field due to elctrons by menas of two scalar potential functions. Proc. Lond. Math. Soc. 1904, 2, 367-372. [CrossRef]

2. Ruse, H.S. On Whittaker's Electromagnetic 'scalar potentials'. Q. J. Math. 1937, 8, 148-160. [CrossRef]

3. Barut, A.O.; Malin, S.; Semon, M. Electrodynamics in terms of functions over the group SU(2): II. Quantization. Found. Phys 1982, 12, 521-530. [CrossRef]

4. Barut, A.O.; Malin, S.; Semon, M. Solution of the basic problems of electrodynamics in the group-space formulation. Il Nuovo Cimento 1985, 89, 64-88. [CrossRef]

5. Friedman, Y.; Gwertzman, S. The scalar complex potential of the electromagnetic field. arXiv 2009, arXiv:0906.0930.

6. Friedman, Y.; Ostapenko, V. The complex pre-potential and the Aharonov-Bohm effect. J. Phys. A Math. Theor. 2010, 43, 405305. [CrossRef]

7. Friedman, Y. The wave-function description of the electromagnetic field. J. Phys. Conf. Ser. 2013, 437, 012018. [CrossRef]

8. Aharonov, Y.; Bohm, D. Significance of Electromagnetic Potentials in the Quantum Theory. Phys. Rev. 1959, 115, 485-491. [CrossRef]

9. Hong, I.K.; Kim, C.S. Quaternion electromagnetism and the relation with two-spinor formalism. Universe 2019, 5, 135-154. [CrossRef]

10. Penrose, R.; Rindler, W. Spinors and Spacetime; Cambridge University Press: Cambridge, UK, 1986 ; Volume 1.

11. Baylis, W.E. Electrodynamics, A Modern Geometric Approach; Progress in Physics 17; Birkhäuser: Boston, MA, USA, 1999.

12. Jackson, J.D. Classical Electrodynamics; Wiley \& Sons: New York, NY, USA, 1999.

13. Friedman. Y.; Danziger, M. The complex Faraday tensor for relativistic evolution of a charged particle in a constant field. PIERS Proc. 2008, 4, 529 -533.

14. Friedman, Y.; Semon, M. Relativistic acceleration of charged particles in uniform and mutually perpendicular electric and magnetic fields as viewed in the laboratory frame. Phys. Rev. E 2005, 72, 026603. [CrossRef]

15. Bliokh, K.; Bekshaev, A.; Nori, F. Dual electromagnetism: Helicity, spin, momentum, and angular momentum. New J. Phys. 2013, 15, 033026. [CrossRef]

16. Drummond, P.D. Dual-symmetric Lagrangians and conservations laws. Phys. Rev. A 1999, 60, 3331-3334. [CrossRef]

17. Drummond, P.D. Dual-symmetric Lagrangians in quantum electrodynamics: I. Conservation laws and multi-polar coupling. J. Phys. B At. Mol. Opt. Phys. 2006 , 39, S573. [CrossRef]

18. Zwanzuger, D. Quantum field theory of particles with both electric and magnetic charges. Phys. Rev. 1968, 176, 1489-1495. [CrossRef]

19. Newman, E.T.; Penrose, R. An Approach to gravitational radiation by a method of spin coefficients. J. Math. Phys. 1962, 3, 566-768. [CrossRef]

20. Jakbsen, H.P.; Vergne, M. Wave and Dirac operators, and representations of the conformal group. J. Funct. Anal. 1977, 24 52-106. [CrossRef]

21. Baylis, W.E.; Cabrera, R.; Keselica, J.D. Quantum/Classical Interface: Classical Geometric Origin of Fermion Spin. Adv. Appl. Clifford Algebr. 2010, 20, 517. [CrossRef]

22. Gersten, A. Maxwell Equations as the One-Photon Quantum Equation. Found. Phys. Lett. 1999, 12, $291-298$. [CrossRef]

23. Friedman, Y.; Scarr, T. Symmetry and Special Relativity. Symmetry 2019, 11, 1235. [CrossRef]

24. Fernandez-Corbaton, I.; Zambrana-Puyalto, X.; Tischler, N.; Vidal, X.; Juan, M.L.; Molina-Teriza, G. Electromagnetic duality symmetry and helicity conservation for the macroscopic Maxwell's equations. Phys. Rev. Lett. 2013, 111, 060401 [CrossRef] [PubMed]

25. Sudbery, A. Quantum Mechanics and the Particles of Nature; Cambridge University Press: Cambridge, UK, 1986; p. 318. 
26. Silberstein, L. Nachtrag zur Abhandlung üher 'elektromagnetische Grundgleichungen in bivektorieller Behandlung'. Ann. Phys. Lpz. 1907, 24, 783-784. [CrossRef]

27. Mashhoon, B. Conformal Symmetry, Accelerated Observers, and Nonlocality. Symmetry 2019, 11, 978. [CrossRef]

(c) (1)

(C) 2019 by the authors. Licensee MDPI, Basel, Switzerland. This article is an open access article distributed under the terms and conditions of the Creative Commons Attribution (CC BY) license (http:/ / creativecommons.org/licenses/by/4.0/). 